\title{
Global stability of a jet in crossflow
}

\author{
SHERVIN BAGHERI ${ }^{1}$, PHILIPP SCHLATTER ${ }^{1}$, \\ PETER J. SCHMID ${ }^{2}$ AND DAN S. HENNINGSON ${ }^{1} \dagger$ \\ ${ }^{1}$ Linné Flow Centre, Department of Mechanics, Royal Institute of Technology (KTH), \\ SE-10044 Stockholm, Sweden \\ ${ }^{2}$ Laboratoire d'Hydrodynamique (LadHyX), CNRS-Ecole Polytechnique, 91128 Palaiseau, France
}

(Received 14 October 2008 and in revised form 12 January 2009)

A linear stability analysis shows that the jet in crossflow is characterized by selfsustained global oscillations for a jet-to-crossflow velocity ratio of 3. A fully threedimensional unstable steady-state solution and its associated global eigenmodes are computed by direct numerical simulations and iterative eigenvalue routines. The steady flow, obtained by means of selective frequency damping, consists mainly of a (steady) counter-rotating vortex pair (CVP) in the far field and horseshoe-shaped vortices close to the wall. High-frequency unstable global eigenmodes associated with shear-layer instabilities on the CVP and low-frequency modes associated with shedding vortices in the wake of the jet are identified. Furthermore, different spanwise symmetries of the global modes are discussed. This work constitutes the first simulation-based global stability analysis of a fully three-dimensional base flow.

\section{Introduction}

The generic flow configuration of a jet in crossflow is ubiquitous in a great variety of industrial applications, ranging from the control of boundary-layer separation to pollutant dispersal from chimneys, from film cooling of turbine blades to the injection of fuel into combustion chambers and furnaces. The flow structures, mixing properties and general dynamics of jets in crossflow have therefore been the subject of numerous experimental and computational studies. In general four main coherent structures (see e.g. Fric \& Roshko 1994; Kelso, Lim \& Perry 1996; Muppidi \& Mahesh 2007 and the references therein) characterize the jet in crossflow: (i) the counter-rotating vortex pair (CVP), which originates in the near field of the jet and essentially follows the jet trajectory and dominates the flow field far downstream; (ii) the shear-layer vortices which are located at the upstream side of the jet and take the form of ring-like or loop-like filaments; (iii) horseshoe vortices forming in the flat-plate boundary layer upstream of the jet exit and corresponding wall vortices downstream of the exit close to the wall; and (iv) 'wake vortices/upright vortices' which are vertically oriented shedding vortices in the wake of the jet. The accurate description of these relevant features is a prerequisite for a sound understanding of the perturbation dynamics of jets in crossflow and a first step in an attempt to manipulate it.

Recent advances in computational methods have enabled global stability analyses of flows with nearly arbitrary complexity and have furnished the possibility to assess fully two- and three-dimensional base flows as to their stability and response behaviour to general three-dimensional perturbations. Specifically, the combination of new efficient 
methods for computing steady-state solutions, such as the selective frequency damping (Åkervik et al. 2006), and for treating very large eigenvalue problems, such as the Arnoldi method implemented in the software package ARPACK (Lehoucq, Sorensen \& Yang 1998), based on only minimal modifications of existing numerical simulation codes (see e.g. Barkley, Gomes \& Henderson 2002) has provided the necessary tools for an encompassing study of the disturbance behaviour in complex flows.

Previous stability investigations of the jet in crossflow (Alves, Kelly \& Karagozian 2007,2008 ) have been based on various inviscid base flows adapted from the vortexsheet model of Coelho \& Hunt (1989); they found that growth rates increase as the jet inflow ratio $R \equiv V_{j e t} / U_{\infty}$ decreases. Recently, Megerian et al. (2007) found experimentally that for a low jet inflow ratio $R<3.5$ external excitations have a small impact on the flow response, in contrast to the significant effect of forcing for larger values of $R$. This indicates a transition from a globally unstable flow in which intrinsic self-sustained global oscillations are present to a convectively unstable flow that exhibits a noise-amplifying behaviour (Huerre 2000). In this paper we analyse the global stability of a jet in crossflow for $R=3$ based on a steady exact solution to the Navier-Stokes equations. Except for preliminary stability studies of a time-averaged mean flow (Schmid 2007), the present analysis constitutes, to the authors' knowledge, the first simulation-based global stability analysis of the viscous jet in crossflow and, more generally, of a fully three-dimensional base flow.

\section{Flow configuration and numerical method}

The stability of a jet in crossflow is analysed via fully resolved direct numerical simulations based on the incompressible Navier-Stokes equations. The computational domain consists of a rectangular box containing the crossflow boundary layer subject to a zero pressure gradient starting at a finite Reynolds number $R e_{\delta_{0}^{*}}$ downstream of the leading edge. All flow quantities are non-dimensionalized using the (constant) free-stream velocity $U_{\infty}$, the viscosity $v$ and the displacement thickness $\delta_{0}^{*}$ at the inlet of the crossflow into the computational box yielding the definition of the Reynolds number as $\operatorname{Re}_{\delta_{0}^{*}} \equiv U_{\infty} \delta_{0}^{*} / \nu$. The two parameters of the jet are the diameter $D$ and the inflow ratio $R \equiv V_{\text {jet }} / U_{\infty}$, where $V_{\text {jet }}$ is the centreline jet velocity. The flow parameters of the present study, $R e_{\delta_{0}^{*}}=165, \delta_{0}^{*} / D=1 / 3$ and $R=3$, are in the same parameter range as those of for example Kelso et al. (1996).

The jet is introduced via non-homogeneous boundary conditions of the wall-normal velocity component at the wall $(y=0)$ at a distance downstream of the inlet. This is in slight contrast to realistic configurations in which the jet emanates from a nozzle, and interactions between the crossflow and the flow near the nozzle edge have been observed (Kelso et al. 1996). The direct numerical simulations of the jet in $\S 3$ demonstrate that even with the jet modelled as an inhomogeneous boundary condition the main flow characteristics such as the CVP, shear-layer vortices and horseshoe vortices are faithfully reproduced. Moreover, the instabilities explored in the present study are most likely due to the interaction of the crossflow and the shear layer further away from the wall (Megerian et al. 2007). The jet profile, mimicking the (laminar) parabolic velocity profile of pipe Poiseuille flow, is imposed as

$$
v(r)=R\left(1-r^{2}\right) \exp \left(-(r / 0.7)^{4}\right),
$$

with $r$ denoting the distance from the jet centre $\left(x_{j e t}, z_{j e t}\right)$, normalized by half the jet diameter $D$. Due to the super-Gaussian function, the profile has continuous derivatives for all $r$ without a large modification of the parabolic shape near the jet centreline. 
(a)

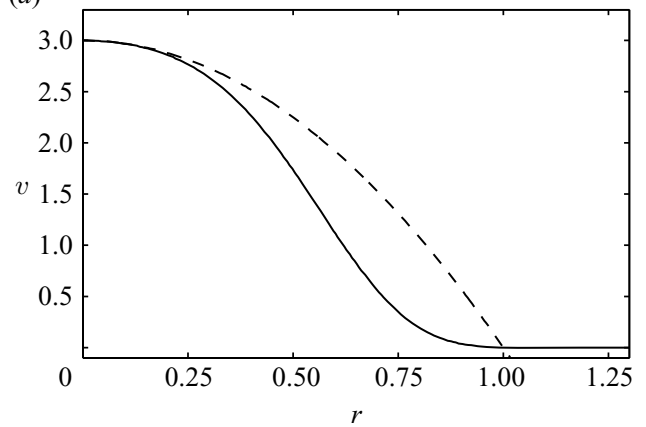

(b)

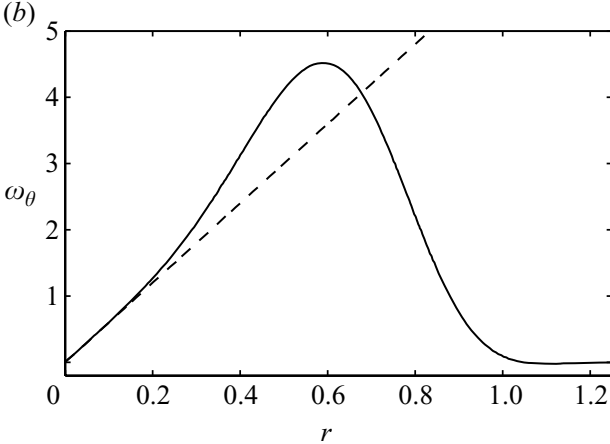

Figure 1. (a) The wall-normal velocity $v$ and $(b)$ the azimuthal vorticity $\omega_{\theta}=-\partial_{r} v$ of the imposed jet profile in the present study given by (2.1) are shown with solid black lines, and those given by the standard Poiseuille parabolic profile are shown with dashed lines. Note that the streamwise velocity $u$ and spanwise velocity $w$ are assumed to be zero everywhere at $y=0$.

The velocity and vorticity of the profile defined by (2.1) are compared to the parabolic profile $\left(R\left(1-r^{2}\right)\right)$ in figure 1 .

The simulation code uses a Fourier-Chebyshev spectral method to solve the threedimensional time-dependent incompressible Navier-Stokes equations over a flat plate, with details given in Chevalier et al. (2007). At the downstream edge of the domain a fringe region is added that forces the flow back to the inlet profile and thus allows the treatment of the streamwise direction by Fourier methods; periodic boundary conditions are imposed in the spanwise direction, whereas in the wall-normal direction no-slip (at $y=0$ ) and Neumann (at $y=L_{y}$ ) conditions are enforced. The computations in this study have been performed with a resolution of $256 \times 201 \times 144$ grid points in a computational box $\left(L_{x}, L_{y}, L_{z}\right)=(75,20,30)$ on a Linux cluster employing between 48 and 72 cores.

\section{Observations from direct numerical simulations}

The present simulation of a jet in crossflow starts with a laminar Blasius profile above a flat plate. At time $t \geqslant 0$, the inhomogeneous boundary condition is imposed to introduce the jet into the computational domain; after approximately 50 time units, a statistically stationary state can be observed. A snapshot at $t=151$ of the flow development is shown in figure 2(a), where isocontours of the $\lambda_{2}$ criterion (Jeong $\&$ Hussain 1995) and the streamwise velocity $u$ are displayed. Although both the boundary layer of the crossflow and the incoming flow pertaining to the jet are laminar, their interaction results in a highly unsteady flow field. At the upstream edge of the jet body, where the shear layer is the strongest, a flow instability develops which leads to the breakdown of the laminar flow into a sequence of small-scale, half-ring-shaped vortices. As these structures convect downstream their orientation gradually aligns with the free stream after which they dissipate due to viscous effects. Careful inspection of the velocity fields of the unsteady direct simulations further reveals well-known flow features as reported in e.g. Fric \& Roshko (1994) and Kelso et al. (1996): Horseshoe-shaped vortices, located inside the crossflow boundary layer, can be detected; they wrap around the nozzle and eventually transport fluid from the outer boundary layer region closer to the wall, resulting in a high-speed region around the plane $z=0$ which widens in the downstream direction (see the grey contours of the $u$ velocity component depicted in figure $2 a$ ). In addition, the core of 

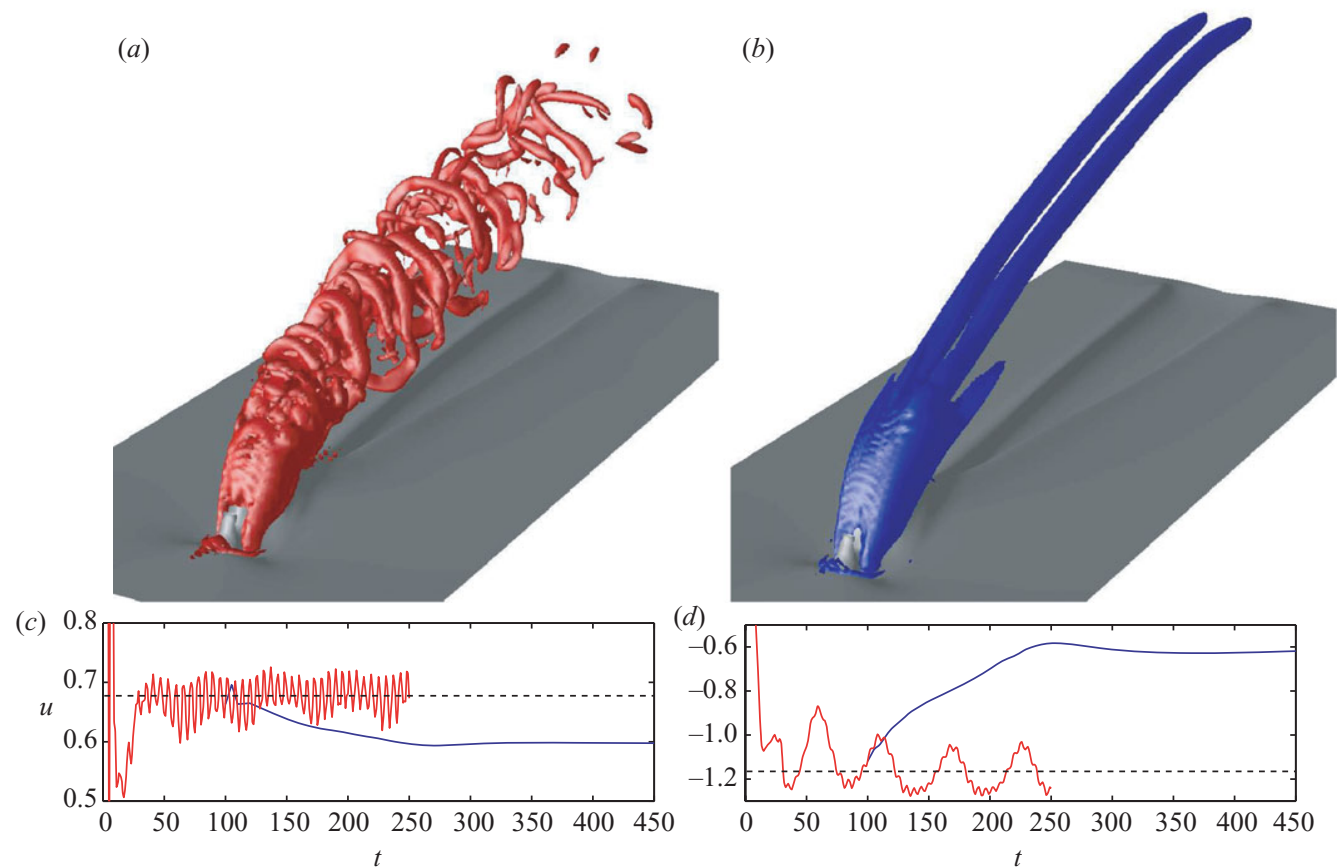

Figure 2. $(a, b)$ The red and blue isocontours correspond to vortical structures visualized by the $\lambda_{2}$ criterion (Jeong \& Hussain 1995) at the level $\lambda_{2}=-0.09$. The grey contours depict the streamwise velocity component $u=0.3$ near the flat plate. (a) Snapshot of the unsteady velocity field in a fully developed state at $t=151$. ( $b$ ) Converged steady base velocity field. $(c, d)$ Time signals corresponding to the (streamwise) $u$ component of two probes in the flow field (see the text). The red line represents the unsteady flow, whereas the blue line corresponds to the simulation stabilized by selective frequency damping which is active for $t>100$. The dashed lines indicate the time-averaged values of the direct numerical simulation.

the jet is composed of two large-scale counter-rotating vortices on which shear-layer instabilities develop. However, wake vortices connecting the crossflow boundary layer and the jet body, reminiscent of a vortex street behind bluff bodies, are not visible in the present simulation. As stated by Fric \& Roshko (1994) these vortices appear preferably at higher inflow ratios $R$ and Reynolds numbers $R e$. For lower $R$ and $R e$, like in our simulation, the spanwise symmetry of the flow field is sustained at all times.

To extract and quantify the observed oscillatory behaviour of the flow we place two probes into the computational domain: one probe is located within the jet shear layer slightly upstream of the jet body, approximately three jet diameters from the wall; the other probe is positioned downstream of the jet orifice close to the wall. After a transient period, the time signals of the streamwise velocity $u$ from these probes clearly show two distinct frequencies $\lambda_{i}$ : in the jet shear layer a period $T_{1}=2 \pi / \lambda_{i} \approx 5.7$ can be estimated (red line in figure $2 c$ ), whereas in the wake of the jet a much longer period of $T_{2} \approx 60$ is present (red line in figure $2 d$ ). The associated Strouhal numbers, defined as $S t=D /\left(T V_{\text {jet }}\right)$, are $S t=0.17$ and $S t=0.016$, respectively. It is interesting to note that the velocity signal recorded by the second probe is negative, i.e. $u \approx-1.1$, which indicates that downstream of the jet exit a region of reversed flow exists. The signal from that region shows oscillations at a rather low frequency. This separation region 
appears to only oscillate in a spanwise-symmetric manner, since the symmetry of the flow field near the jet exit is not broken, given our parameter settings. The frequency measured in the jet shear layer corresponds to the incipient vortical structures caused by the presence of the incoming jet. However, even in the shear layer the lower frequency present in the wake downstream of the nozzle is clearly felt, manifesting itself as a slow modulation of the probe signals. This indicates that the entire jet is oscillating with that long period $T_{2}$.

\section{Global stability analysis}

Using the direct numerical simulation code described in $\S 2$ a global stability analysis can be performed in two steps: we first compute a steady solution to the nonlinear Navier-Stokes equations using the selective frequency damping method, after which we determine the eigenmodes of Navier-Stokes equations linearized about this base flow using the Arnoldi algorithm from the parallel ARPACK library.

\subsection{The three-dimensional base flow}

When a flow under consideration is either globally unstable or strongly convectively unstable, the computation of a steady-state solution of the Navier-Stokes equations poses a challenging task. Newton iteration methods are often applied, but a different approach that accomplishes the task with less programming effort is favoured here. This technique, known as selected frequency damping (Åkervik et al. 2006), adds a forcing term

$$
-\chi(\boldsymbol{u}-\hat{\boldsymbol{u}})
$$

to the right-hand side of the Navier-Stokes equations governing the evolution of the flow $\boldsymbol{u}=(u, v, w)$. This results in the convergence towards a temporally low-passfiltered state $\hat{\boldsymbol{u}}$ of the nonlinear equations using the differential form of an exponential (causal) filter (Pruett et al. 2003),

$$
\partial_{t} \hat{\boldsymbol{u}}=(\boldsymbol{u}-\hat{\boldsymbol{u}}) / \Delta .
$$

The filter coefficients have to be chosen to sufficiently damp the lowest unstable frequencies; information about these frequencies can straightforwardly be extracted from the simulations (see figure $2 c, d$ ). The same figure also shows the effect of the filtering, turned on at $t=100$ for $\chi=1$ and $\Delta=2$. Moreover, we could observe that after 220 time units, $\left\|\partial_{t} \boldsymbol{u}\right\|$ decays at an exponential rate, and at $t=450$ the norm is three orders of magnitude smaller than the unsteady flow, yielding a sufficiently converged steady solution of the nonlinear Navier-Stokes equations and thus our three-dimensional base flow (denoted $\boldsymbol{U}$ hereafter).

The base flow is shown in figure $2(b)$ with blue $\left(\lambda_{2}\right)$ and grey $(u)$ contour levels. The CVP is evident as two distinct tubes of negative $\lambda_{2}$ isocontours, deflected along the jet trajectory and slowly decaying in the streamwise direction. Physically, the appearance of the CVP can be explained by the accumulation of the azimuthal vorticity related to the injected jet fluid, which is redirected in the direction of the jet trajectory due to the interaction with the crossflow (Fric \& Roshko 1994). Additionally, the steady-state features the horseshoe-shaped vortex forming around the jet nozzle. Due to this vortex, a small region of separated flow appears just upstream of the jet nozzle. Moreover, a larger region with negative streamwise velocity is detected at the downstream edge of the nozzle, causing entrainment of fluid from the crossflow into the jet. 

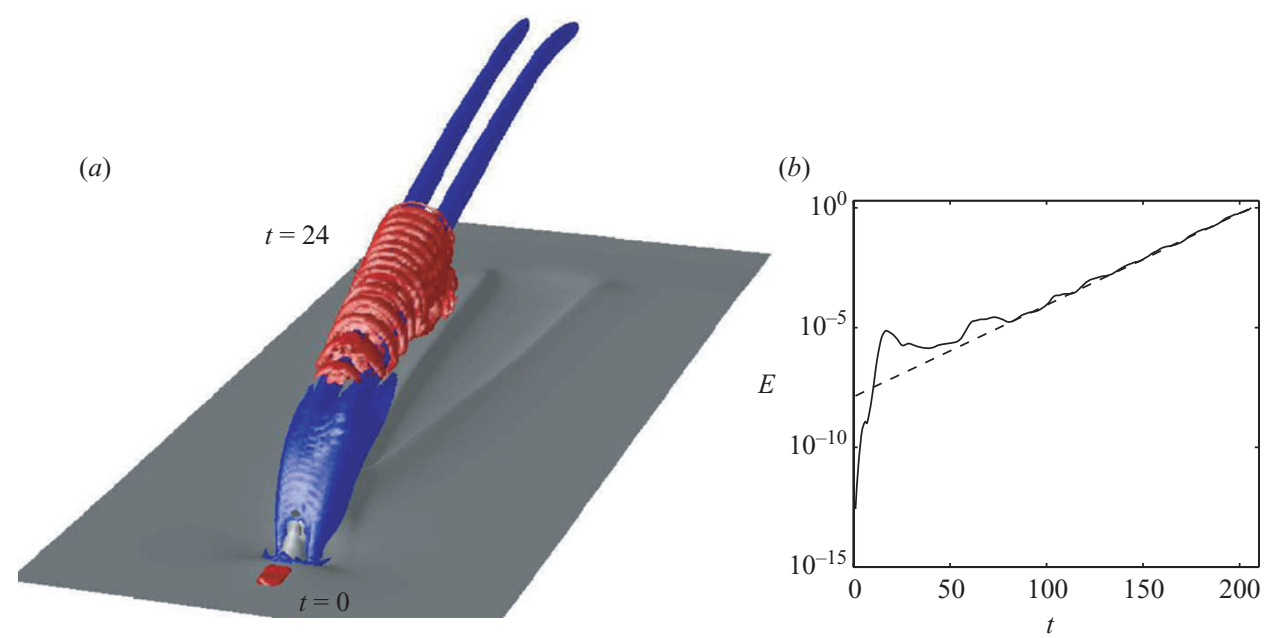

FIGURE 3. (a) The two structures depicted with red $\lambda_{2}$ isocontours represent a symmetric initial perturbation upstream of the jet at $t=0$ and the perturbation at later time, $t=24$. The grey and blue isocontours indicate components of the steady base flow as described in the caption of figure 2. (b) The time evolution of the perturbation energy (solid line) is compared to the growth rate (dashed line) of the most unstable symmetric global mode, marked with $S_{3}$ in figure $4(a, c)$.

By construction ( $\AA$ 2006), the converged solution obtained by SFD satisfies the steady nonlinear Navier-Stokes equations. Alternatively, a linear stability analysis can be performed of the time-averaged mean flow. However, this flow is not an equilibrium of the Navier-Stokes equations and, therefore, not a suitable choice for a base flow when the aim is to determine its stability. The time-averaged mean flow may, however, for certain flow configurations, be appropriate as a base flow for predicting the dominant shedding frequencies (Barkley 2006; Sipp \& Lebedev 2007). The difference between steady and time-averaged solutions is exemplified in figure $2(b, c)$, in which the time signals of the probes pertaining to the time-averaged solution (black dashed lines) are clearly different from those obtained using the steady solution (solid blue lines).

A first numerical experiment probing the global stability or instability of the steady flow consists of an initial Gaussian pulse released at $t=0$ inside the boundary layer just upstream of the jet exit (indicated with red $\lambda_{2}$ contour levels in figure $3 a$ ). The linear response of the base flow after 24 time units, also shown in figure 3(a), is characterized by the formation of a wave packet which significantly grows in amplitude as it travels along the curved base flow. Quantitative information about the growth rate (figure $3 b$ ) and local wavenumber of this wave packet can easily be extracted from the simulation data, but a more general stability analysis is performed next using a 'time-stepper' approach which extracts the global modes from direct numerical simulations.

4.2. Stability analysis using the Arnoldi method and a time-stepper approach The stability of $\boldsymbol{U}$ is determined by the three-dimensional global modes,

$$
\boldsymbol{\phi}_{j}(x, y, z) \exp \left(\lambda_{j} t\right), \quad j=1, \ldots, m,
$$


of the linearized Navier-Stokes operator, denoted here by the matrix $\boldsymbol{A}$. Both the eigenmodes $\boldsymbol{\phi}_{j}$ and the eigenvalues $\lambda_{j}$ are in general complex valued. The temporal growth rate is given by the imaginary part $\lambda_{j, i}$ and the frequency by the real part $\lambda_{j, r}$.

The size of our problem $\left(\boldsymbol{A}\right.$ would be approximately a $10^{7} \times 10^{7}$ matrix) prohibits matrix methods and suggests the use of iterative techniques combined with our numerical simulations. In general, the iterative technique is based on the orthogonal projection of the large matrix onto a lower-dimensional subspace, which will result in a significantly smaller system that can be solved using direct methods. A particularly useful subspace is the Krylov subspace $\mathscr{K}$ spanned by snapshots taken from flow fields $\boldsymbol{u}$ separated by a constant time interval $\Delta t$,

$$
\begin{aligned}
\mathscr{K} & =\operatorname{span}\{\boldsymbol{u}(\boldsymbol{x}, 0), \boldsymbol{u}(\boldsymbol{x}, \Delta t), \boldsymbol{u}(\boldsymbol{x}, 2 \Delta t), \ldots, \boldsymbol{u}(\boldsymbol{x},(m-1) \Delta t)\} \\
& =\operatorname{span}\left\{\boldsymbol{u}(\boldsymbol{x}, 0), \boldsymbol{B} \boldsymbol{u}(\boldsymbol{x}, 0), \boldsymbol{B}^{2} \boldsymbol{u}(\boldsymbol{x}, 0), \ldots, \boldsymbol{B}^{m-1} \boldsymbol{u}(\boldsymbol{x}, 0)\right\},
\end{aligned}
$$

where $\boldsymbol{B}=\exp (\boldsymbol{A} \Delta t)$ and $\boldsymbol{u}(\boldsymbol{x}, 0)$ is an initial guess that should contain non-zero components of the sought-after global modes. The matrix exponential $\boldsymbol{B}$ is simply an evolution operator; its action is a numerical simulation of the linearized NavierStokes equations for time $\Delta t . \dagger$ Note that the eigenmodes of $\boldsymbol{B}$ are the same as those of the system matrix $\boldsymbol{A}$ if $\Delta t$ is chosen properly, i.e. so that it reflects the characteristic time scale of the physical structures in the flow. More specifically, the choice $\Delta t$ is a balance between the time scale given by the Nyquist criterion $\ddagger$ and a sufficient temporal separation of the Krylov vectors to ensure convergence of the iterative method.

The Krylov subspace is orthonormalized (standard $L_{2}$ vector norm) via an $m$-step Arnoldi factorization, yielding an unitary basis $\boldsymbol{V}$ onto which the matrix exponential is projected according to $\boldsymbol{B} \approx \boldsymbol{V} \boldsymbol{H} \boldsymbol{V}^{T}$, leading to a small $m \times m$ eigenvalue problem of the form $\boldsymbol{H S}=\boldsymbol{\Sigma} \boldsymbol{S}$. The eigenmodes with the largest growth rates are recovered according to $\boldsymbol{\Phi}=\left(\boldsymbol{\phi}_{1}, \ldots, \boldsymbol{\phi}_{m}\right)=\boldsymbol{V} \boldsymbol{S}$, and the eigenvalues $\lambda_{j}$ are given by $\lambda_{j}=\ln \left(\sigma_{j}\right) / \Delta t$ with $\boldsymbol{\Sigma}=\operatorname{diag}\left(\sigma_{1}, \ldots, \sigma_{m}\right)$

The computation of the significantly lower-dimensional matrix $\boldsymbol{H}$ from direct numerical simulations is accomplished by the implicitly restarted Arnoldi method (IRAM) implemented in the software package ARPACK. The number of flow field snapshots separated by $\Delta t=0.25$ required for convergence of the 22 eigenmodes with the largest growth rates was 1800 . However, thanks to IRAM, the Krylov subspace is only of the order $m \approx 60$, and the Arnoldi procedure is restarted with a new improved initial guess, $\boldsymbol{u}(\boldsymbol{x}, 0)$, repeatedly until convergence. The first initial guess was random noise. See e.g. Lehoucq et al. (1998) for details on IRAM.

The residual was $\left\|\boldsymbol{B} \boldsymbol{\phi}_{j}-\sigma_{j} \boldsymbol{\phi}_{j}\right\|<10^{-6}$ for all eigenmodes, although the residuals of most of the eigenmodes are orders of magnitude smaller than this. $\emptyset$

$\dagger$ The actual time step $\delta t \approx 10^{-3}$ of these simulations depends on the CFL condition and is much smaller than the sampling period $\Delta t$.

$\ddagger$ To avoid aliasing $\Delta t$ must be small enough such that two sampling points in one period of the highest frequency mode are obtained. See Bagheri et al. (2009) for further numerical details.

If Within the time-stepping framework, the linear stability analysis is robust with respect to the spatial resolution if it is, as in our case, based on fully resolved direct numerical simulations. A convergence test was performed in the following way: The direct numerical simulation with $\approx 50 \%$ fewer grid points of the linear response of the base flow to an initial condition resulted in a growth rate that was only $\approx 2 \%$ different from the growth rate predicted by the original resolution, albeit the significant reduction in resolution. 

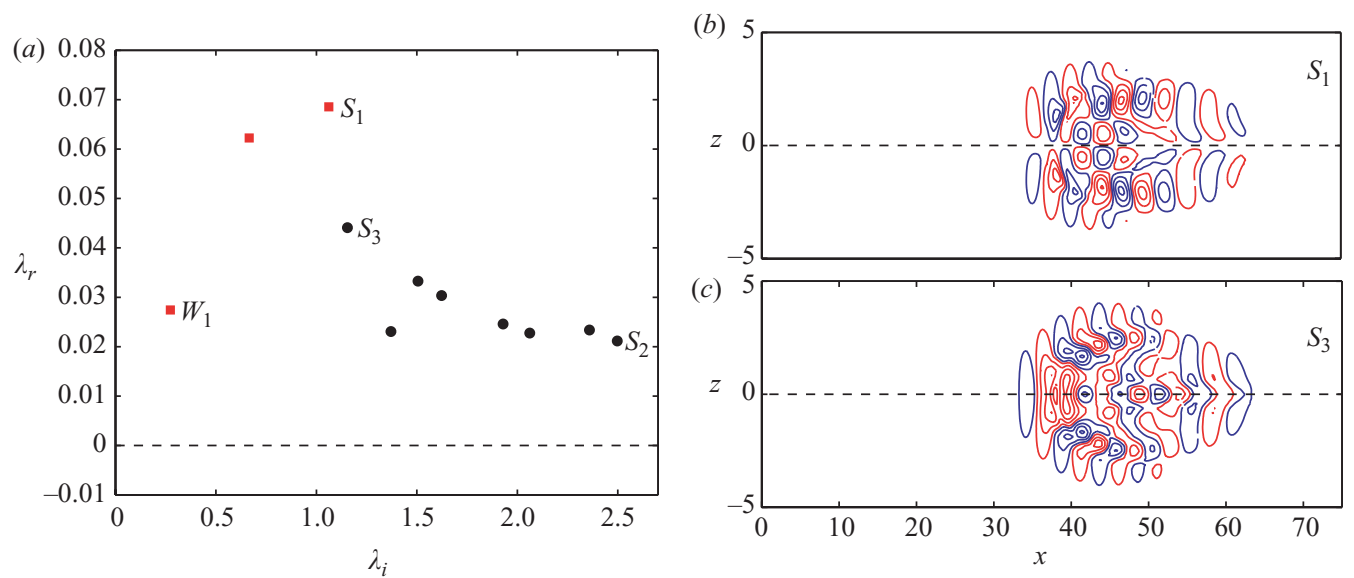

Figure 4. (a) The spectrum of the jet in crossflow, where $\lambda_{r}$ is the growth rate and $\lambda_{i}$ the frequency. High-frequency eigenvalues marked with black circles have symmetric eigenmodes, whereas the red squares have antisymmetric ones. The shear-layer modes marked with $S_{1}$ and $S_{2}$ are shown in figures 5 and 6 , respectively. The wake mode marked with $W_{1}$ is shown in figure 7, and the most unstable symmetric mode marked with $S_{3}$, triggered by the response of the base flow to a symmetric initial condition, is shown in figure 3. $(b, c)$ Contour levels in the $x z$-plane with $y=17$ of the $u$ velocity component(blue negative, red positive) of (b) $S_{1}$ and (c) $S_{3}$.

\subsection{Global spectrum and global modes}

In figure $4(a)$ the global spectrum containing the 11 most unstable eigenvalues is shown. The global eigenmodes corresponding to eigenvalues marked with circles are symmetric with respect to the $z$-axis, whereas the modes associated with eigenvalues marked with squares are antisymmetric. Note that the symmetry refers to the $u$ and $v$ velocity components; $w$ shows the opposite symmetry. It should be mentioned that no symmetry condition was imposed in the direct numerical simulations. The streamwise velocity of two modes $\left(S_{1}\right.$ and $\left.S_{3}\right)$ with opposite symmetries are shown figure $4(b, c)$. A symmetric disturbance in the flow (as shown in figure $3 a$ ) will trigger the growth of a global mode with the same symmetry only. This is exemplified in figure $3(b)$, where the solid line shows the time evolution of the energy $E=\int \boldsymbol{u}^{T} \boldsymbol{u} \mathrm{d} \boldsymbol{x}$ of a symmetric initial perturbation, and the dashed line shows the energy of most unstable symmetric global mode with the growth rate, $\lambda_{r}=0.044$ marked with $S_{3}$ in the spectrum (figure $4 a$ ). This serves as a further validation that the spectrum provides the correct growth rates.

In figure 5 , red $\lambda_{2}$ isocontours pertaining to the most unstable (antisymmetric) mode, oscillating with a period of $T=5.9$ time units, are shown together with the base flow (blue and grey isocontours) as described earlier in figure $2(b)$. The mode takes the shape of a wave packet located on the CVP with a distinct spatial wavelength and can therefore be associated with instabilities rising on the shear layer. The amplitude near the wall of this mode is more than one order of magnitude smaller than on the CVP. It is known that the jet width extends more towards the leeward side. It is interesting to note that the the global mode in figure 5 also contains vortical structures in this region.

Next, a symmetric high-frequency mode is shown in figure 6. The mode is a localized wave packet wrapped around the CVP without any vortical structures close to the wall. The other symmetric modes have a similar structure but with larger wavelengths 
(a)

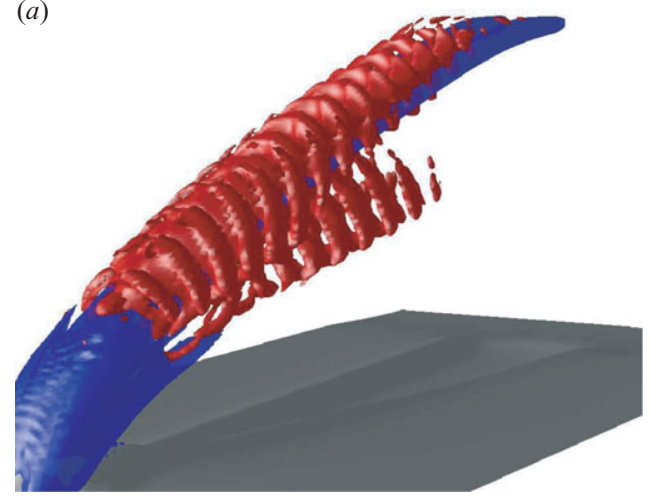

(b)

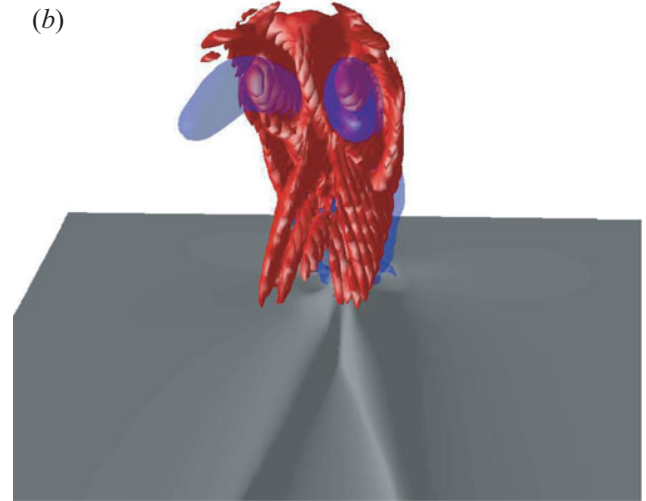

Figure 5. The most unstable mode $\left(\left(\lambda_{r}, \lambda_{i}\right)=(0.068,1.06)\right)$ seen from two different angles, marked with $S_{1}$ in figure 4 , is shown with red $\lambda_{2}$ isocontours. The base flow is shown in blue $\left(\lambda_{2}\right)$ and grey $(u)$.

(a)

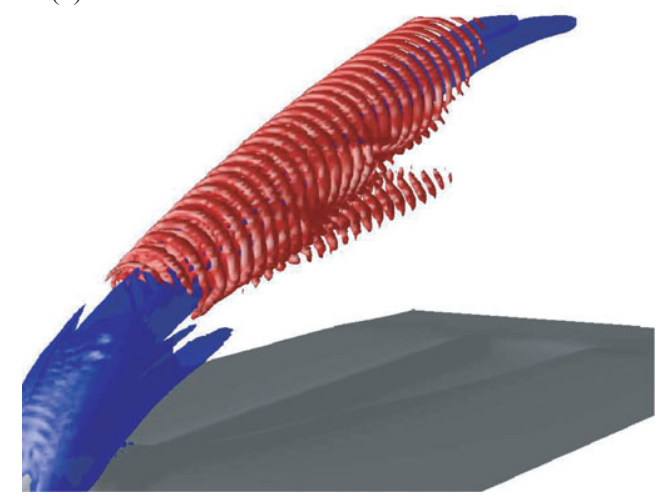

(b)

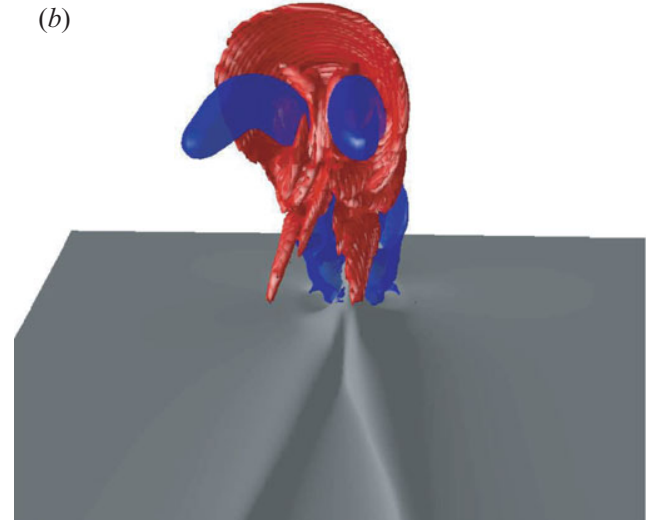

FiguRE 6. The mode with the highest frequency $\left(\left(\lambda_{r}, \lambda_{i}\right)=(0.021,2.49)\right)$ viewed from two different angles, marked with $S_{2}$ in figure 4 , is shown with red $\lambda_{2}$ isocontours and base flow in blue $\left(\lambda_{2}\right)$ and grey $(u)$.

and lower frequencies. In particular the nonlinear shedding of the shear-layer vortices with $T=5.7$ is closely matched by the linear frequency of the most unstable symmetric mode $\left(S_{3}\right) T=5.4$. It is interesting to note that the most unstable shear-layer mode has an opposite symmetry to the all the high-frequency shear-layer modes. Whereas the latter modes are wrapped around the CVP, the former is directly located on the CVP, as can be seen by comparing figures $5(b)$ and $6(b)$.

The vortical structures near the wall become more pronounced with decreasing temporal frequencies. The spanwise velocity component near the wall (green and black contours) of the antisymmetric mode with the lowest frequency $\lambda_{i}$ is shown in figure 7. Of all the modes, this global mode has the most pronounced spatial structure at the wall in the wake of the jet. It is thus assumed that this mode, which oscillates at a period of $T=23$ time units, is related to the low-frequency self-sustained oscillations observed in our direct numerical simulation (see figure $2 d$ ). However, this unstable wake mode has a smaller growth rate than the shear-layer mode, indicating the dominance of the shear-layer instabilities in the present base flow. 


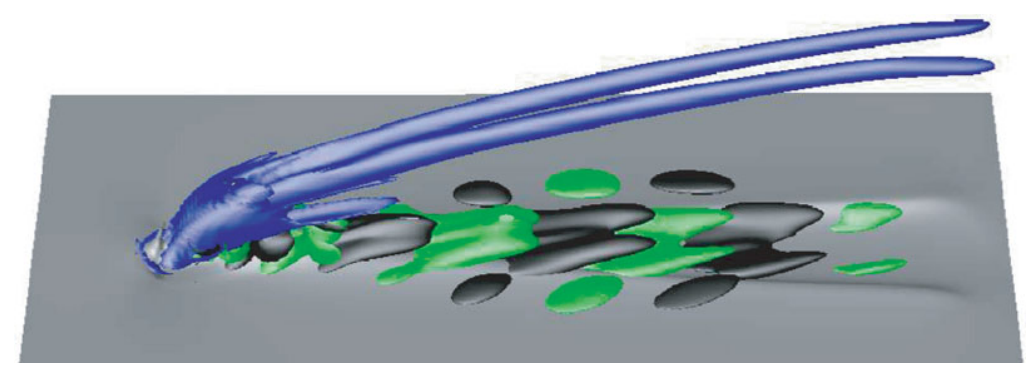

FIGURE 7. Isocontours of the spanwise velocity component pertaining to the low-frequency mode $\left(\left(\lambda_{r}, \lambda_{i}\right)=(0.027,0.27)\right)$, marked $W_{1}$ in the spectrum (figure 4$)$, are shown in green (negative $w$ ) and black (positive $w$ ). Note that here only the structures near the wall are shown, which are approximately one order of magnitude smaller in amplitude than the structures on the CVP. The base flow is again in blue $\left(\lambda_{2}\right)$ and grey $(u)$.

From the numerical simulation, we could detect that close to the wall, approximately 2-3 jet diameters from the nozzle along the jet trajectory, shear-layer vortices were shed periodically into the downstream direction. The largest amplitude of the global eigenmodes, on the other hand, is concentrated further downstream on the jet trajectory of the base flow. This spatial separation of the linear global modes (downstream) and the shedding region (upstream) has been observed for various globally unstable flows (Chomaz 2005) and can be explained by the presence of a 'wavemaker'. From a local viewpoint this indicates that a pocket of absolute instability feeds regions of convective instability located further downstream. From a global viewpoint, which is more suitable due to the non-parallel nature of the base flow, this region is related to the location at which the spatial overlap of the global mode with its associated adjoint mode is significant. A localized feedback force of the perturbation velocity in this region results in the largest drift of the eigenvalue (Giannetti \& Luchini 2007). Moreover, using the adjoint eigenmodes, a sensitivity analysis can be employed to predict regions in which variations of the unstable eigenvalues to base-flow modifications are large (Marquet, Sipp \& Jacquin 2008). Further analysis is in progress to identify these dynamically important regions.

In general, linear (and weakly nonlinear) stability theory is able to predict shedding frequencies in the unstable regime close to the critical velocity ratio. A large deviation between the linear and nonlinear saturated frequencies can to some extent be related to a large difference between the time-averaged mean flow and the steady solution, i.e. the mean flow distortion (see the recent work by Barkley 2006; Sipp \& Lebedev 2007). By comparing the signals in figure $2(c, d)$ pertaining to the mean (dashed) and steady flow (blue), it can be observed that the mean flow distortion near the wall is at least six times larger than on the shear layer. Similar to observations of the wake behind a cylinder (Barkley 2006), the steady separation region (downstream of the jet exit) is altered due to the saturated global modes yielding a significantly larger separation bubble and mean flow distortion. The jet shear layer on the other hand is only slightly modified by the saturated modes.

\section{Conclusions}

Observations of two self-sustained synchronized oscillations of a jet in crossflow at $R=3$ by direct numerical simulations places this flow configuration into the category 
of globally unstable flows. This observation has been confirmed by a global stability analysis based on a three-dimensional steady base state obtained after suppressing global instabilities by selective frequency damping. The most unstable global modes with high frequencies are compact and represent localized wave packets on the CVP. These modes are associated with the loop-shaped vortical structures on the jet shear layer. The global modes with lower frequencies, on the other hand, also have a significant amplitude in the wake of the jet close to the wall and can be associated with less pronounced vortical structures arising downstream of the jet in the boundary layer.

The existence of global eigenmodes justifies the global stability approach as an appropriate tool to describe the inherent and dominant dynamics of the jet in crossflow. With the same tool even less pronounced instabilities, e.g. associated with the 'hanging vortices' formed in the skewed mixing layers on the lateral edges of the jet (Yuan, Street \& Ferziger 1999), might be detected and extracted from the direct numerical simulations as global modes with growth rates much less than the dominant one presented in this paper. A more encompassing global stability analysis of the jet in crossflow, including a parameter study, is in progress; the presented results, however, form a first and important step in quantifying the stability characteristics of a jet in crossflow and, in general, of fully three-dimensional base flows.

The authors acknowledge funding from the Swedish Research Council (VR) and computer-time allocation from the Swedish National Infrastructure for Computing (SNIC). PJS is grateful for funding from the 'Chaires d'excellence' programme of the Agence Nationale de la Recherche (ANR).

\section{REFERENCES}

Åkervik, E., Brandt, L., Henningson, D. S., Heepffner, J., Marxen, O. \& Schlatter, P. 2006 Steady solutions of the Navier-Stokes equations by selective frequency damping. Phys. Fluids 18 (068102), 1-4.

Alves, L., Kelly, R. \& Karagozian, A. 2007 Local stability analysis of an inviscid transverse jet. J. Fluid Mech. 581, 401-418.

Alves, L., Kelly, R. \& Karagozian, A. 2008 Transverse-jet shear-layer instabilities. Part 2. Linear analysis for large jet-to-crossflow velocity ratio. J. Fluid Mech. 602, 383-401.

Bagheri, S., Åkervik, E., Brandt, L. \& Henningson, D. S. 2009 Matrix-free methods for the stability and control of boundary layers. AIAA J. Accepted.

BARKLEY, D. 2006 Linear analysis of the cylinder wake mean flow. Europhys. Lett. 75, 750-756.

Barkley, D., Gomes, M. G. \& Henderson, R. D. 2002 Three-dimensional instability in flow over a backward-facing step. J. Fluid Mech. 473, 167-190.

Chevalier, M., Schlatter, P., Lundbladh, A. \& Henningson, D. S. 2007 SimsON: a pseudospectral solver for incompressible boundary layer flows. Tech Rep. TRITA-MEK 2007:07. KTH Mechanics.

Chomaz, J. M. 2005 Global instabilities in spatially developing flows: non-normality and nonlinearity. Annu. Rev. Fluid Mech. 37, 357-392.

Coelho, S. \& Hunt, J. 1989 The dynamics of the near field of strong jets in crossflows. J. Fluid Mech. 200, 95-120.

Fric, T. F. \& Roshko, A. 1994 Vortical structure in the wake of a transverse jet. J. Fluid Mech. 279, $1-47$.

GiannetTi, F. \& LuChini, P. 2007 Structural sensitivity of the first instability of the cylinder wake. J. Fluid Mech. 581, 167-197.

Huerre, P. 2000 Open shear flow instabilities. (ed. G. K. Batchelor, H. K. Mofatt and M. G. Worster) In Perspectives in Fluid Dynamics, pp. 159-229. Cambridge University Press.

Jeong, J. \& Hussain, F. 1995 On the identification of a vortex. J. Fluid Mech. 285, 69-94. 
Kelso, R., Lim, T. \& Perry, A. 1996 An experimental study of round jets in cross-flow. J. Fluid Mech. 306, 111-144.

LehoucQ, R., Sorensen, D. \& YAng, C. 1998 ARPACK Users' Guide: Solution of Large-Scale Eigenvalue Problems with Implicitly Restarted Arnoldi Methods. SIAM.

Marquet, O., SipP, D. \& JACQUin, L. 2008 Sensitivity analysis and passive control of cylinder flow. J. Fluid Mech. 615, 221-252.

Megerian, S., Davitian, L., Alves, L. \& Karagozian, A. 2007 Transverse-jet shear-layer instabilities. Part 1. Experimental studies. J. Fluid Mech. 593, 93-129.

Muppidi, S. \& MAhesh, K. 2007 Direct numerical simulation of round turbulent jets in crossflow. J. Fluid Mech. 574, 59-84.

Pruett, C. D., Gatski, T. B., Grosch, C. E. \& Thacker, W. D. 2003 The temporally filtered Navier-Stokes equations: properties of the residual stress. Phys. Fluids 15 (8), 2127-2140.

Schmid, P. J. 2007 Nonmodal stability theory. Annu. Rev. Fluid Mech. 39, 129-162.

SipP, D. \& Lebedev, A. 2007 Global stability of base and mean flows: a general approach and its applications to cylinder and open cavity flows. J. Fluid Mech. 593, 333-358.

YuAn, L. L., Street, R. L. \& Ferziger, J. H. 1999 Large-eddy simulation of a round jet in crossflow. J. Fluid Mech. 379, 71-104. 